\title{
Eficácia do sistema WaveOne Gold no preparo de canais longo ovais com instrumentos únicos e em modo sequencial
}

\author{
Effectiveness of the WaveOne Gold system in preparing long oval canals with unique instruments \\ and in sequential mode
}

Eficacia del sistema WaveOne Gold en la preparación de canales ovalados largos con instrumentos únicos y en modo secuencial

Wander José da Silva

ORCID: https://orcid.org/0000-0002-5613-5081

Universidade Estadual de Campinas, Brasil

E-mail: wanderjose@fop.unicamp.br

Luiz Fernando Fariniuk

ORCID: https://orcid.org/0000-0003-0731-9893 Instituto Odontológico das Américas, Brasil

E-mail: fariniuk@uol.com.br

Lara Dalla Vecchia Beira

ORCID: https://orcid.org/0000-0003-3121-1292

Universidade da Região de Joinville, Brasil

E-mail: lara.dalla.vecchia.beira@hotmail.com

Flares Baratto-Filho

ORCID: https://orcid.org/0000-0002-5649-7234

Universidade da Região de Joinville, Brasil

E-mail: fbaratto1@gmail.com

Marilisa Carneiro Leão Gabardo

ORCID: https://orcid.org/0000-0001-6832-8158 Universidade Positivo, Brasil

E-mail: marilisagabardo@gmail.com

\begin{abstract}
Resumo
O objetivo deste estudo foi avaliar variação de volume, porcentagem de áreas não instrumentadas e de debris após o preparo de canais longo ovais com o sistema WaveOne ${ }^{\circledR}$ Gold (WOG) com duas técnicas: uso cada instrumento dos sistema (single-file - SF) e uso de todas as limas do sistema de modo sequencial (multiple-file - MF). Foram selecionados 50 incisivos inferiores humanos, distribuídos em cinco grupos $(\mathrm{n}=10)$. Após verificação da dimensão radicular com limas manuais, todos os espécimes foram submetidos à microtomografia computadorizada $(\mu \mathrm{CT})$ para análise de variação de volume, porcentagem de áreas não instrumentadas e de debris após o preparo. Os espécimes foram preparados com instrumentos WOG, de modo SF e MF. Os dados foram analisados quanto à normalidade e homogeneidade de variância. Conforme o desfecho, foram aplicados os testes de One-Way ANOVA seguido de Games-Howell ou Tukey, e Kruskal-Wallis seguido de Dunn. Todos os grupos apresentaram variação de volume total
\end{abstract}


e independentemente da técnica utilizada, o terço cervical teve maior ampliação quando comparado ao apical ( $\mathrm{p}<$ 0.05). Em relação à porcentagem de áreas não instrumentadas e de debris foram observadas diferenças significativas para WOG Medium versus WOG Small a WOG Medium $(\mathrm{p}<0.05)$. Ambas as técnicas, SF e MF, proporcionaram variação de volume após o preparo, com persistência de áreas não instrumentadas e de debris. Contudo, melhores resultados ocorerram quando houve maior ampliação do canal radicular.

Palavras-chave: Raios x; Microtomografia por raio-x; Endodontia; Preparo do canal radicular.

\begin{abstract}
The aim of this study was to evaluate variation in volume, percentage of non-instrumented areas and debris after preparing long oval canals with the WaveOne ${ }^{\circledR}$ Gold (WOG) system with two techniques: using each instrument of the system (single-file - SF) or use of all files in the system sequentially (multiple-file - MF). Fifty lower human incisors were selected, distributed in five groups $(\mathrm{n}=10)$. After verification of the root dimension with manual files, all specimens were submitted to microcomputed tomography $(\mu \mathrm{CT})$ for analysis of volume variation, percentage of non-instrumented areas and debris after preparation. The specimens were prepared with WOG instruments, using SF and MF mode. The data were analyzed for normality and homogeneity of variance. Depending on the outcome, OneWay ANOVA tests followed by Games-Howell or Tukey, and Kruskal-Wallis followed by Dunn were applied. All groups showed a variation in total volume and regardless of the technique used, the cervical third had greater magnification when compared to the apical one $(\mathrm{p}<0.05)$. Regarding the percentage of non-instrumented areas and debris, significant differences were observed for WOG Medium versus WOG Small to WOG Medium ( $\mathrm{p}<0.05$ ). Both techniques, SF and MF, provided volume variation after preparation, with persistence of non-instrumented areas and debris. However, better results occur when there has been greater enlargement of the root canal.
\end{abstract}

Keywords: X-ray; X-ray microtomography; Endodontics; Root canal preparation.

\title{
Resumen
}

El objetivo de este estudio fue evaluar la variación en volumen, porcentaje de áreas no instrumentadas y detritos luego de preparar largos conductos ovalados con el sistema WaveOne ${ }^{\circledR}$ Gold (WOG) con dos técnicas: usando cada instrumento del sistema (single-file - SF ) o el uso de todos los archivos del sistema de forma secuencial (varios archivos - MF). Se seleccionaron cincuenta incisivos humanos inferiores, distribuidos en cinco grupos $(\mathrm{n}=10)$. Después de la verificación de la dimensión de la raíz con archivos manuales, todas las muestras se sometieron a tomografía microcomputada $(\mu \mathrm{CT})$ para el análisis de la variación de volumen, el porcentaje de áreas no instrumentadas y los desechos después de la preparación. Las muestras se prepararon con instrumentos WOG, utilizando los modos SF y MF. Los datos se analizaron para determinar la normalidad y la homogeneidad de la varianza. Dependiendo del resultado, se aplicaron las pruebas ANOVA unidireccionales seguidas de Games-Howell o Tukey, y Kruskal-Wallis seguidas de Dunn. Todos los grupos mostraron una variación en el volumen total e independientemente de la técnica utilizada, el tercio cervical tuvo mayor aumento en comparación con el apical (p $<0.05$ ). Con respecto al porcentaje de áreas no instrumentadas y escombros, se observaron diferencias significativas para WOG Medium versus WOG Small a WOG Medium ( $\mathrm{p}<0.05$ ). Ambas técnicas, SF y MF, proporcionaron variación de volumen después de la preparación, con persistencia de áreas no instrumentadas y detritos. Sin embargo, se obtienen mejores resultados cuando ha habido un mayor agrandamiento del conducto radicular.

Palabras clave: Rayos X; Microtomografía por rayos X; Endodoncia; Preparación del conducto radicular.

\section{Introdução}

Embora o debridamento do sistema de canais radiculares, por meio do preparo químico-mecânico, seja considerado passo fundamental na terapia endodôntica (Hülsmann et al., 1997; Schilder, 1974), sabe-se que há apenas uma redução de microrganismos nessa etapa (Baratto-Filho et al., 2009; Guillén et al., 2018). A literatura demonstra que tal condição é influenciada pela variabilidade anatômica interna dos dentes, especialmente aqueles com canais ovais ou achatados (BarattoFilho et al., 2009, Busquim et al. 2015; Langeland et al., 1985; Versiani et al., 2011; Sousa-Neto et al., 2018).

Os incisivos inferiores são dentes que apresentam características de achatamento radicular devido à maior dimensão no sentido vestíbulo-lingual (Milzanezi de Almeida et al., 2013; Wu et al., 2000). Essa característica favorece a ocorrência de áreas não instrumentadas (De-Deus et al., 2015; Espir et al., 2018), bem como acúmulo de debris contaminados (De-Deus et al., 2015; Paqué et al., 2009; Versiani et al., 2016). Nesses casos, melhores resultados para desinfecção têm sido encontrados de modo proporcional à maior ampliação do canal radicular (Lorencetti et al., 2014; Plotino et al., 2019). Entretanto, deve-se considerar que o desgaste excessivo das paredes dentinárias, decorrente do uso de instrumentos circulares e calibrosos, pode levar ao enfraquecimento da raiz (Wu et al., 2000). 
Assim, novos sistemas e técnicas têm sido introduzidos no mercado continuamente com intuito de aprimorar o preparo dos canais radiculares. Atualmente, a maioria se apresenta como instrumentos de uso único, com a proposta de diminuição de custo e tempo operatório (Yared, 2008). O sistema WaveOne ${ }^{\circledR}$ Gold (WOG) surge nesse contexto com quatro instrumentos com conicidade/taper diferentes: Small (20/.07) - WS, Primary (25/.07) - WP, Medium (35/.06) - WM e Large (45/.05) - WL. Sua evolução em relação ao sistema WaveOne ${ }^{\circledR}$ caracteriza-se pela liga utilizada no processo de fabricação (NiTi) com tratamento térmico (Gold wire), conferindo ao instrumento maior flexibilidade, resistência à fadiga cíclica, segurança e eficiência de corte (van der Vyver \& Vorster, 2017).

Para análise do preparo dos canais radiculares, a literatura é vasta quanto à indicação da microtomografia computadorizada $(\mu \mathrm{CT}$ ) (Espir et al., 2018; Plotino et al., 2006; Villas-Bôas et al., 2011). Além de ser uma técnica não destrutiva, demonstra o impacto do preparo e oferece ao pesquisador resultados quantitativos, que possibilitam comparações pré e pós instrumentação (Alencar et al., 2008), tornando cada espécime seu próprio controle (Nielsen et al, 1995). A técnica permite, ainda, o equilíbrio tridimensionalmente da amostra (Sousa-Neto et al., 2018). Quanto à diversidade de análises com uso desse método, encontram-se variação de volume, porcentagem de áreas não instrumentadas e presença de debris no canal radicular (De-deus et al. 2015; Versiani et al. 2016).

O presente estudo teve como objetivo analisar o preparo de canais longo ovais com sistema WOG, com protocolos single- file (SF) ou multiple-file (MF). A hipótese nula testada foi que as diferentes técnicas não influenciam os desfechos testados: volume do canal radicular, porcentagem de áreas não instrumentadas e de debris.

\section{Metodologia}

Para o cálculo amostral dados de estudos prévios foram utilizados como referência (De-Deus et al., 2019; Velozo et al., 2020) e realização do cálculo do poder observado na amostra, sendo considerado o $\alpha=5 \%$, o que resultou em um valor de $99 \%$.

Após aprovação do Comitê de Ética (registro n. ${ }^{\circ}$ 2.420.082), 50 incisivos inferiores foram selecionados. Os critérios de inclusão foram: canal radicular único, rizogênese completa, ausência de tratamento endodôntico e calcificações, reabsorções interna e externa, dilacerações ou fraturas. Para a caracterização dos espécimes como longo ovais foi aplicada a classificação de $\mathrm{Wu}$ et al. (2000). Os selecionados foram mantidos em solução de cloreto de sódio $(\mathrm{NaCl})$ a 0,9\% (Eurofarma, São Paulo, Brasil) até o início do experimento, com substituição da solução a cada 7 dias.

Inicialmente as coroas foram seccionadas com disco diamantado dupla face (KG Sorensen, Cotia, Brasil) para padronização das raízes em um comprimento de $15 \mathrm{~mm}$. A patência de todos os canais radiculares foi estabelecida com uma lima tipo K \#10 (Dentsply Sirona Endodontics, Ballaigues, Suíça), no comprimento de trabalho (CT) de $14 \mathrm{~mm}$ (1 mm aquém do comprimento real do dente). Os ápices radiculares foram então isolados com resina composta (Filtek ${ }^{\circledR}$ Z350; 3M ESPE, Saint Paul, EUA).

Uma aproximação com a atividade clínica, como proposta por van der Vyver e Vorster (2017), que fizeram uso de limas manuais para explorar os canais e assim escolheram o instrumento adequado para o preparo, foi aqui adotada. Em seguida, a distribuição dos quatro grupos amostrais foi realizada de acordo com os calibres $0,20 \mathrm{~mm}(\mathrm{n}=12), 0,25 \mathrm{~mm}(\mathrm{n}=$ 11), 0,35 mm ( $\mathrm{n}=15)$ e $0,45 \mathrm{~mm}(\mathrm{n}=12)$. Os espécimes foram explorados com limas tipo $\mathrm{K} \# 10, \mathrm{~K} \# 15$, Flexofile $(\mathrm{FF}) \# 20 \mathrm{e}$ FF \#25 (Dentsply Sirona Endodontics, Ballaigues, Suíça). Os sobressalentes dessa divisão ( $\mathrm{n}=10)$ foram aleatorizados em um quinto grupo de preparo, o MF.

Neste estudo o sistema WOG foi testado em duas formas: instrumento único -SF e instrumento em modo sequencial MF. Na SF cada espécime foi preparado com apenas um instrumento (WS, WP, WM ou WL) de acordo com o diâmetro apical inicial (D0); na MF o preparo foi realizado com uso sequencial de todo o sistema WOG. 
Para o preparo, os instrumentos utilizados foram acoplados ao motor elétrico X-Smart Plus ${ }^{\circledR}$ (Dentsply Sirona Endodontics, Ballaigues, Suíça), e os movimentos executados foram de "bicada", com amplitude de 3mm, no sentido coroaápice até que o CT fosse atingido. A irrigação dos espécimes para todos os grupos foi feita com seringa descartável de $5 \mathrm{ml}$ acoplada a uma ponta irrigadora com agulha de $30 \mathrm{G}$ (Navitip; Ultradent, South Jordan, EUA), calibrada a $3 \mathrm{~mm}$ aquém do CT (11 mm). Foram utilizados $2 \mathrm{ml}$ de $\mathrm{NaOCl} 2.5 \%$ a cada avanço de $3 \mathrm{~mm}$ do instrumento no interior do canal radicular. Entre cada avanço foi verificada a patência com lima $\mathrm{K} \# 10$, até ser atingido o $\mathrm{CT}$. No total, todos os espécimes foram irrigados com $8 \mathrm{ml}$ de $\mathrm{NaOCl} 2.5 \%+10 \mathrm{ml}$ EDTA 17\% + $2.5 \mathrm{ml}$ de NaOCl, conforme protocolo de Plotino et al. (2019).

Toda parte experimental foi realizada por um único operador, endodontista com 5 anos de experiência. Neste estudo cada instrumento foi utilizado em três espécimes, conforme encontrado na literatura (Bueno et al., 2017; Gergi et al., 2014, 2015; Lim et al., 2013; Tambe et al., 2014).

As amostras foram digitalizadas pré e pós preparo com uso de um microtomógrafo (SkyScan 1174; Bruker-microCT, Kontich, Bélgica), sob os parâmetros de $52 \mathrm{kV}, 790 \mu \mathrm{A}$, com tempo de exposição de aproximadamente 48 minutos, voxel isotrópico (tamanho do pixel) de $19,76 \mu \mathrm{m}$, giro de $0.8^{\circ}$ e filtro de $0.5 \mathrm{~mm}$ Al. Inicialmente a reconstrução das radiografias foi realizada pelo software NRecon (Bruker-microCT, Kontich, Bélgica) e o pareamento das mesmas em relação à altura foi realizado no Data Viewer (Bruker-microCT Kontich, Bélgica). Todas as imagens foram reconstruídas a partir do ápice radicular até a junção amelocementária, originando 450 secções (fatias), para análise por terços cada espécime foi dividido em: cervical (região I), médio (região II) e apical (região III), a partir da divisão do número total de fatias tomográficas por três. Em cada terço, 150 secções foram selecionadas para as diferentes variáveis.

Pelo software CTAn v.1.12.0.0 (Bruker-microCT, Kontich, Bélgica), foi criado o processo de renderização da fase binarizada, para aquisição de parâmetros quantitativos do canal, bem como caracterização da anatomia interna de acordo com a classificação de Wu et al. (2000). Pelo CTVol (Bruker-microCT, Kontich, Bélgica) foi reproduzido o modelo em 3D, e assim foi possível ser destacada uma fase binarizada da outra.

Para análise de volume pré e pós preparo foi calculada a variação dos dados ( $\Delta=$ volume final - volume inicial). A análise da porcentagem de áreas não instrumentadas foi realizada pelo cálculo da divisão do número de voxels estáticos (presentes na mesma posição na superfície radicular antes e após a instrumentação), dividido pelo número total de voxels na superfície do canal (Paqué \& Peters, 2011). A presença de debris foi expressada pela intersecção entre as imagens pré e pós instrumentação (De-Deus et al., 2014; Paqué et al., 2009; Robinson et al., 2012).

Após análise descritiva inicial, os dados foram submetidos aos testes de normalidade (Shapiro-Wilk) e de homogeneidade de variância (Levene). Para a variação do volume total foram empregados os testes One-Way ANOVA seguido de Games-Howell. Quando em questão os terços, foi aplicado Kruskal-Wallis seguido do pós-teste de Dunn. Para a porcentagem total de áreas não instrumentadas realizado teste de Kruskal-Wallis, seguido do pós-teste de Dunn; para análise por terços, aplicou-se One-Way ANOVA seguido de teste de Tukey. Por fim, a porcentagem total e por terços, de debris, foi analisada pelos testes de Kruskal-Wallis e de Dunn.

Todas as análises foram realizadas em SPSS (IBM ${ }^{\circledR}$ Statistics v. 25.0, SPSS Inc, Chicago, EUA), com adoção de nível de significância de 5\%.

\section{Resultados}

Houve homogeneidade amostral $(\mathrm{p}=0,101)$, o que indicou que a alocação dos espécimes nos grupos amostrais foi adequada. 
Para a variação de volume, por terços, diferenças significativas foram observadas na técnica SF na região cervical apenas para WL quando comparado aos demais ( $\mathrm{p}<0.05)$; no terço médio WS foi igual a WP ( $\mathrm{p}=0.290)$, mas diferiu dos outros ( $<$ 0.05); no terço apical diferiram WS e WL ( $\mathrm{p}=0.039)$ (Tabela 1). A técnica MF não apresentou resultados significativos nas comparações múltiplas $(\mathrm{p}>0.05)$ na comparação por terços.

Tabela 1 - Variação do volume $(\Delta)\left(\mathrm{mm}^{3}\right)$ por terços (mediana: mínimo-máximo) e total (média: \pm DP).

\begin{tabular}{lcccc}
\hline \multirow{2}{*}{ Grupos } & \multicolumn{3}{c}{ Terços } & \multirow{2}{*}{ Total } \\
\cline { 2 - 4 } WS & \multicolumn{1}{c}{ Cervical } & Médio & Apical & $1,47(0,77)^{\mathrm{C}}$ \\
\cline { 2 - 4 } WP & $0,83(0,21-2,99)^{\mathrm{B}}$ & $0,45(0,11-0,81)^{\mathrm{B}}$ & $0,08(-0,02-0,35)^{\mathrm{B}}$ & $1,72(1,36)^{\mathrm{B}, \mathrm{C}}$ \\
WM & $0,24(0,09-2,69)^{\mathrm{B}}$ & $0,76(0,37-0,86)^{\mathrm{A}, \mathrm{B}}$ & $0,08(-0,18-0,13)^{\mathrm{A}, \mathrm{B}}$ & $2,28(0,91)^{\mathrm{B}, \mathrm{C}}$ \\
WL & $0,91(0,20-1,32)^{\mathrm{B}}$ & $0,95(0,67-2,29)^{\mathrm{A}}$ & $0,33(0,14-0,93)^{\mathrm{A}, \mathrm{B}}$ & $3,49(0,59)^{\mathrm{A}, \mathrm{B}}$ \\
WS a WP & $1,77(0,71-2,71)^{\mathrm{A}}$ & $1,22(0,74-1,69)^{\mathrm{A}}$ & $0,58(0,46-0,82)^{\mathrm{A}}$ & $4,68(2,16)^{\mathrm{A}, \mathrm{B}}$ \\
WS a WM & $2,35(0,64-5,29)^{\mathrm{A}}$ & $1,32(0,78-2,86)^{\mathrm{A}}$ & $0,61(-0,01-0,90)^{\mathrm{A}, \mathrm{B}}$ & $3,53(0,79)^{\mathrm{A}, \mathrm{B}}$ \\
WS a WL & $2,37(1,26-3,74)^{\mathrm{A}}$ & $1,16(0,47-1,78)^{\mathrm{A}}$ & $0,41(0,18-0,64)^{\mathrm{A}, \mathrm{B}}$ & $4,09(1,28)^{\mathrm{A}}$ \\
\hline
\end{tabular}

Nota: Letras maiúsculas diferentes indicam diferença estatisticamente significativa na coluna. Letras minúsculas indicam diferença estatisticamente significativa na linha.

*Kruskal-Wallis seguido de teste post hoc de Dunn $(\mathrm{p}<0.05)$.

***ne-Way ANOVA seguido de teste post hoc de Games-Howell $(\mathrm{p}<0.05)$.

Fonte: Autores.

Na comparação entre técnicas foram observadas diferenças significativas apenas no terço cervical para WP versus WS a WP (p = 0,007), e WM versus WS a WM (p = 0,004) (Tabela 1).

Acerca da variação do volume total, para a técnica SF, apenas WS versus WL diferiram ( $\mathrm{p}<0.001)$. Já na MF não ocorreram diferenças entre os grupos ( $p$ > 0.05), bem como na comparação entre técnicas em relação ao referido instrumento, por exemplo, WP versus WS a WP (Tabela 1).

A avaliação das áreas não instrumentadas demonstrou menor porcentagem dessas em relação à maior ampliação do canal radicular (Tabela 2). Quando considerados os terços, a região cervical na técnica SF apresentou diferença entre WS versus WL ( $\mathrm{p}<00.01$ ); nos terços médio e apical, WM e WL foram iguais entre si ( $\mathrm{p}>0.05)$, mas diferentes de WS ( $\mathrm{p}<0.05)$. Não houve diferença significativa na técnica MF ( $p$ > 0.05) nas mesmas comparações. Para a porcentagem total de áreas não instrumentadas, constatou-se diferença significativa na técnica SF para WL quando comparada aos demais grupos $(\mathrm{p}<0.01)$, bem como nas comparações entre técnicas para WM versus WS a WM $(\mathrm{p}<0.002)$. Nas comparações entre grupos e intragrupos, na técnica MF, não foram encontradas diferenças significativas ( $\mathrm{p}>0.05)$. Pela sobreposição de imagens pré e pós preparo foram identificadas regiões não instrumentadas no canal radicular (Figuras 1 e 2). 
Research, Society and Development, v. 10, n. 5, e53010515500, 2021

(CC BY 4.0) | ISSN 2525-3409 | DOI: http://dx.doi.org/10.33448/rsd-v10i5.15500

Tabela 2 - Porcentagem de áreas não instrumentadas por terços (média: \pm DP) e total (mediana: mínimo-máximo).

\begin{tabular}{lcccc}
\hline \multirow{2}{*}{ Grupos } & \multicolumn{2}{c}{ Terços* } & \multirow{2}{*}{ Total** } \\
\cline { 2 - 4 } & Cervical & Médio & Apical & (1) \\
WS & $52,48(14,17)^{\mathrm{B}}$ & $58,40(11,09)^{\mathrm{B}}$ & $66,63(14,56)^{\mathrm{B}}$ & $56,93(31,74-86,33)^{\mathrm{A}}$ \\
WP & $52,87(18,58)^{\mathrm{A}, \mathrm{B}}$ & $35,82(11,21)^{\mathrm{A}, \mathrm{B}}$ & $45,38(13,52)^{\mathrm{A}, \mathrm{B}}$ & $11,84(0,00-73,80)^{\mathrm{A}, \mathrm{B}}$ \\
WM & $34,65(10,04)^{\mathrm{A}, \mathrm{B}}$ & $35,01(10,39)^{\mathrm{A}}$ & $37,39(13,40)^{\mathrm{A}}$ & $34,91(0,00-53,14)^{\mathrm{A}, \mathrm{B}}$ \\
WL & $23,59(11,04)^{\mathrm{A}}$ & $24,38(13,10)^{\mathrm{A}}$ & $18,09(11,64)^{\mathrm{A}}$ & $18,01(4,95-46,51)^{\mathrm{C}}$ \\
WS a WP & $17,02(13,09)^{\mathrm{A}}$ & $20,06(13,99)^{\mathrm{A}}$ & $38,89(11,90)^{\mathrm{A}}$ & $21,62(0,86-53,30)^{\mathrm{B}, \mathrm{C}}$ \\
WS a WM & $15,26(14,19)^{\mathrm{A}}$ & $19,33(14,96)^{\mathrm{A}}$ & $23,04(15,18)^{\mathrm{A}}$ & $16,26(1,18-49,19)^{\mathrm{C}}$ \\
WS a WL & $13,67(13,60)^{\mathrm{A}}$ & $14,85(13,19)^{\mathrm{A}}$ & $21,62(14,47)^{\mathrm{A}}$ & $12,52(0,26-52,46)^{\mathrm{C}}$ \\
\hline
\end{tabular}

Nota: Letras maiúsculas diferentes indicam diferença estatisticamente significativa na coluna.

*One-Way ANOVA seguido de teste post hoc de Tukey $(\mathrm{p}<0.05)$.

**Kruskal-Wallis seguido de teste post hoc de Dunn $(\mathrm{p}<0.05)$.

Fonte: Autores. 
Figura 1. Sobreposição das imagens pré e pós preparo por terços e total na técnica SF. Imagem tridimensional reconstruída de incisivos inferiores representativa para os grupos WS, WP, WM e WL (eixo horizontal). (A) reconstruções pré-operatórias; (B) reconstruções pós-operatórias; (C) reconstruções sobrepostas; (D) seções transversais para os terços cervical, médio e apical dos canais. Vermelho indica área pré-operatória; verde indica área pós-operatória.
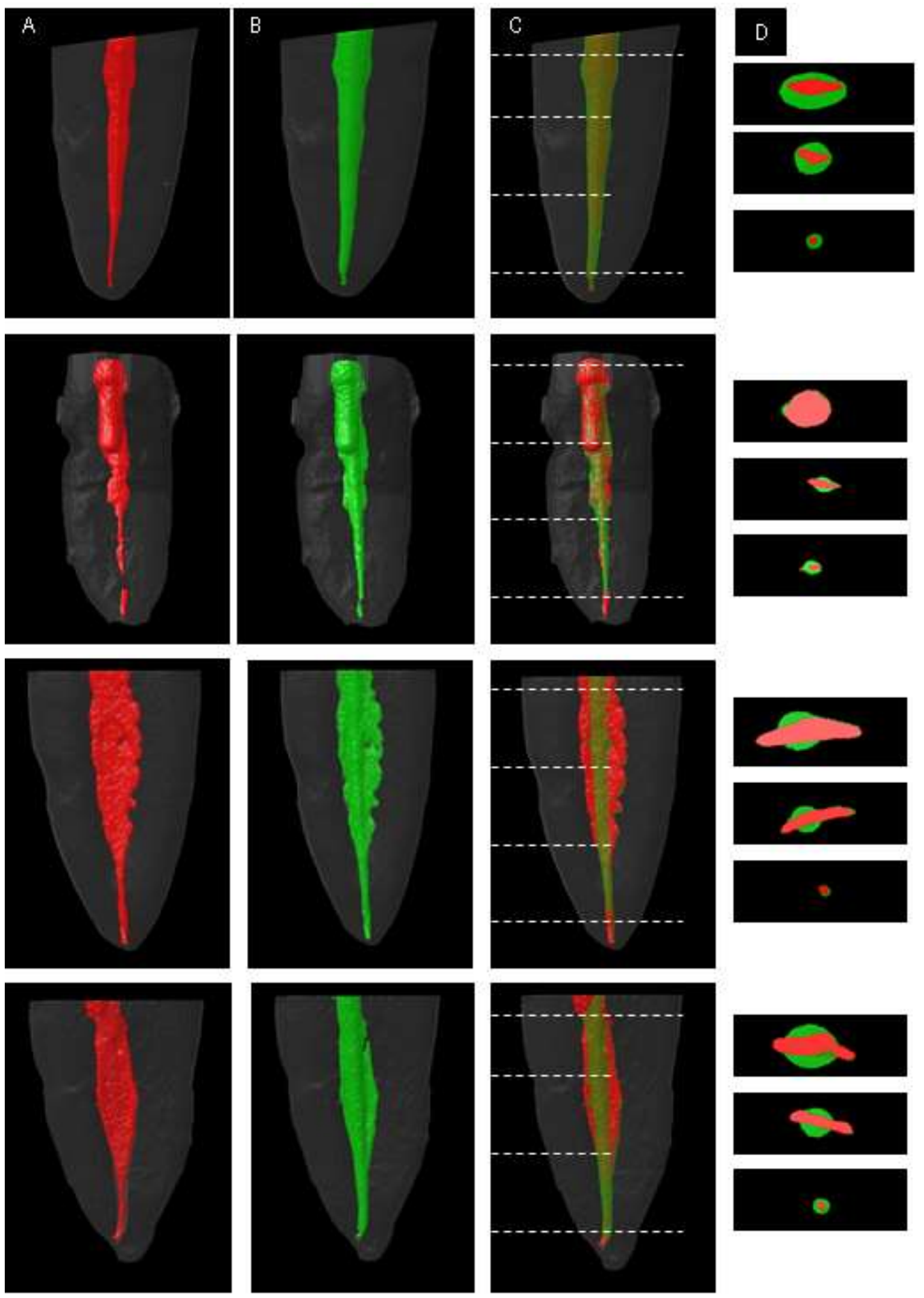

Fonte: Autores.
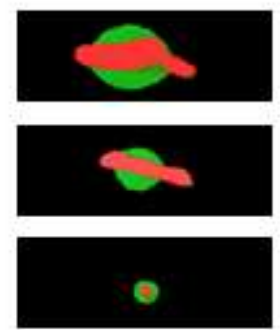
Figura 2. Sobreposição das imagens pré e pós preparo por terços e total na técnica MF. Imagem tridimensional reconstruída de incisivos inferiores representativa para o grupo WS a WL (eixo vertical). (A) reconstruções pré-operatórias; (B) reconstruções pós-operatórias grupo WS; (C) reconstruções pós-operatórias grupo WS a WP; (D) reconstruções pós-operatórias grupo WS a WM e (E) reconstruções pós-operatórias grupo WS a WL. Vermelho indica área pré-operatória; verde indica área pósoperatória.
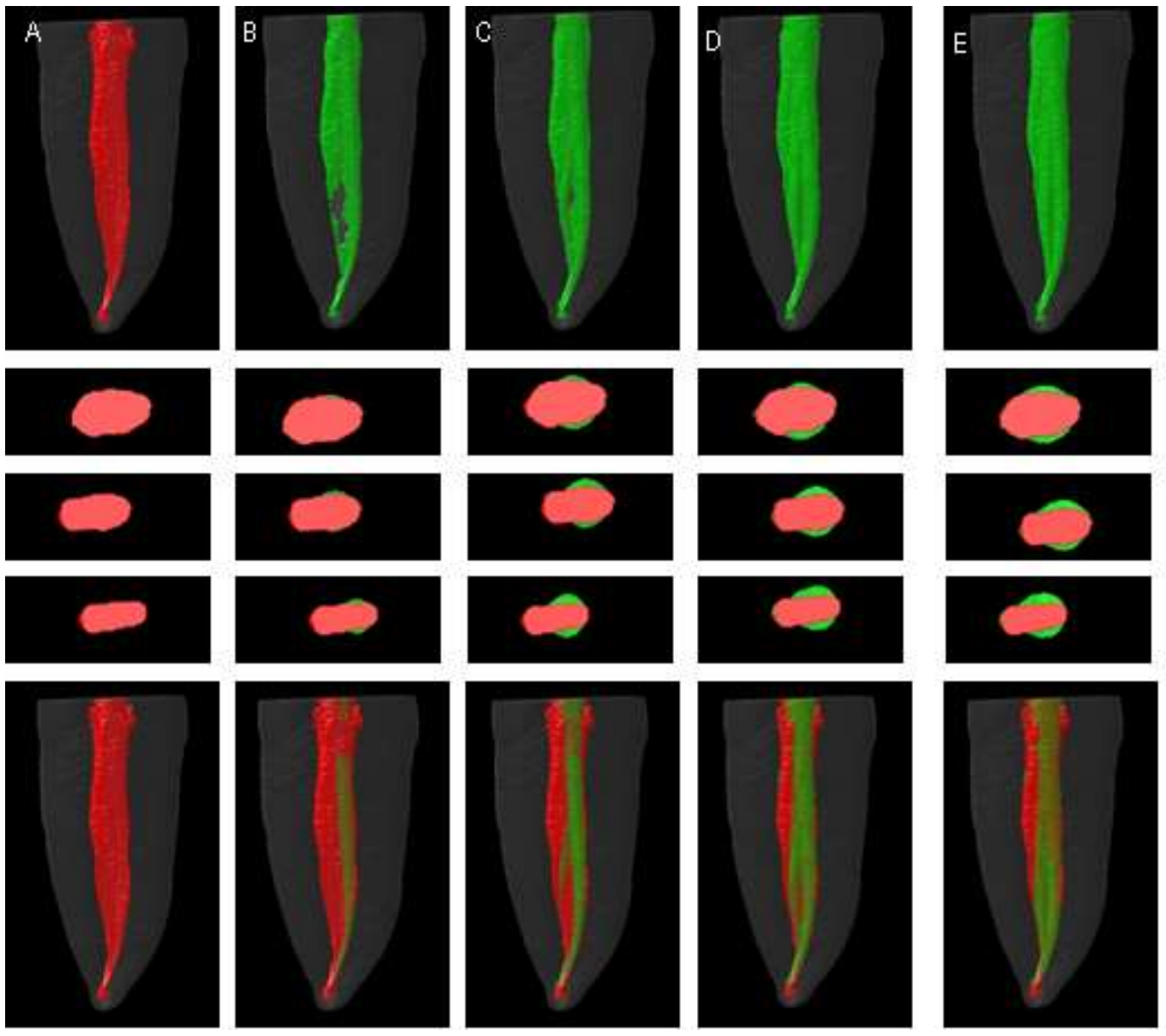

Fonte: Autores.

Notou-se menor porcentagem de debris proporcionalmente à maior ampliação do canal radicular. Considerando a análise por terços e total, melhores resultados foram encontrados para WL na técnica SF nos três terços $(p<0.01)$, enquanto na técnica MF não houve diferenças significativas ( $\mathrm{p}>0.05$ ). Em SF e MF diferenças aconteceram entre WM versus WS a WM ( $\mathrm{p}<0.01)$. Na comparação entre terços, no mesmo grupo, foram encontradas diferenças significativas $(\mathrm{p}>0,05)$ (Tabela 3$)$. 
Tabela 3 - Porcentagem de debris por terços e total (mediana: mínimo-máximo).

\begin{tabular}{|c|c|c|c|c|}
\hline \multirow{2}{*}{ Grupos } & \multicolumn{4}{|c|}{ Terços } \\
\hline & Cervical & Médio & Apical & Total \\
\hline WS & $0,21(0,11-1,69)^{\mathrm{B}}$ & $0,26(0,03-1,06)^{\mathrm{B}}$ & $0,17(0,03-0,39)^{\mathrm{B}}$ & $0,50(0,11-2,76)^{\mathrm{A}}$ \\
\hline WP & $0,19(0,07-0,50)^{\mathrm{A}, \mathrm{B}}$ & $0,04(0,03-0,88)^{\mathrm{A}, \mathrm{B}}$ & $0,03(0,01-0,49)^{\mathrm{A}, \mathrm{B}}$ & $0,20(0,00-1,22)^{\mathrm{B}, \mathrm{C}}$ \\
\hline WM & $0,05(0,02-0,33)^{\mathrm{A}, \mathrm{B}}$ & $0,20(0,02-0,47)^{\mathrm{A}}$ & $0,12(0,00-0,64)^{\mathrm{A}}$ & $0,37(0,00-0,86)^{\mathrm{A}, \mathrm{C}}$ \\
\hline WL & $0,05(0,02-0,40)^{\mathrm{A}}$ & $0,05(0,00-0,09)^{\mathrm{A}}$ & $0,02(0,01-0,07)^{\mathrm{A}}$ & $0,12(0,03-0,72)^{\mathrm{B}, \mathrm{C}, \mathrm{D}}$ \\
\hline WS a WP & $0,02(0,00-0,31)^{\mathrm{A}}$ & $0,02(0,00-0,40)^{\mathrm{A}}$ & $0,04(0,01-0,26)^{\mathrm{A}}$ & $0,85(0,03-0,87)^{\mathrm{B}, \mathrm{D}}$ \\
\hline WS a WM & $0,01(0,01-0,31)^{\mathrm{A}}$ & $0,01(0,00-0,26)^{\mathrm{A}}$ & $0,00(0,00-0,13)^{\mathrm{A}}$ & $0,04(0,00-0,72)^{\mathrm{B}, \mathrm{D}}$ \\
\hline WS a WL & $0,01(0,00-0,27)^{\mathrm{A}}$ & $0,01(0,00-0,33)^{\mathrm{A}}$ & $0,01(0,00-0,11)^{\mathrm{A}}$ & $0,02(0,00-0,71)^{\mathrm{D}}$ \\
\hline
\end{tabular}

Nota: Letras maiúsculas diferentes indicam diferença estatisticamente significativa na coluna.

*Kruskal-Wallis seguido de teste post hoc de Dunn ( $\mathrm{p}<0,05)$.

Fonte: Autores.

\section{Discussão}

Neste estudo propôs-se avaliar o desempenho do sistema WOG, em modo SF e MF, no preparo de canais longo ovais. Os resultados apontaram variação de volume após o preparo em todos os casos, com MF sendo superior até mesmo na capacidade de tocar áreas do canal radicular e remover debris, o que rejeita a hipótese nula testada.

A técnica de preparo do canal radicular com uso de apenas um instrumento de níquel-titânio, sob cinemática reciprocante, foi proposta por Yared (2008). Desde então, diversos instrumentos vêm sendo introduzidos continuamente no mercado com a proposta de simplificar a instrumentação dos canais radiculares (van der Vyver \& Vorster, 2017). Apesar desses avanços, a literatura ainda demonstra a persistência de áreas não instrumentadas após o preparo, que abrigam debris contaminados, o que favorece o insucesso do tratamento endodôntico, pois bactérias remanescentes nessas áreas são potencialmente responsáveis pela inflamação periapical persistente (Versiani et al., 2016). Na tentativa de serem superadas possíveis limitações do preparo com instrumento único, na presente pesquisa empregou-se para alguns grupos uma técnica de preparo com uso do sistema completo, aqui denominado MF.

Atualmente a efetividade do preparo de canais radiculares tem sido descrita por meio da variação de volume, com análises dos efeitos da instrumentação do canal pela remoção da dentina (Gergi et al., 2015; Neves et al., 2016; Schilder, 1974). Neste estudo, o sistema WOG apresentou maior aumento de volume quando utilizada a técnica MF, mas sem ter havido diferença significativa para SF. Estudos apontam que, quando em análise canais longo ovais, os sistemas BioRace e TRUShape não demonstraram diferença significativa para aumento de volume quando comparados a Reciproc e Self-Adjusting File (SAF) (Zuolo et al., 2018). Diferenças também não foram observadas por Versiani et al. (2018) com os sistemas XP-endo Shaper, iRaCe e EdgeFile, e por Guimarães et al. (2017) com TRUShape e Reciproc. Ambos os estudos demonstraram aumento de volume, sem diferença significativa entre os instrumentos e técnicas utilizadas, corroborando os achados aqui encontrados.

Quanto ao aumento de volume do canal radicular por terços, na região cervical houve diferença significativa para WL na técnica SF, e entre técnicas (SF e MF) para WP e WM, quando comparados WS a WP e WS a WM, respectivamente. O maior aumento na região cervical está relacionado à maior conicidade e diâmetro do instrumento que atua nessa área. Para a variação da região apical não foram observadas diferenças significativas, corroborando os achados de Guimarães et al. (2017), onde apenas a região apical foi avaliada. Embora a padronização do preparo apical (40/.06) difira do presente estudo, com variadas conicidades, essa similaridade de resultados pode ser explicada pela diferença no processo de formação dos grupos experimentais. De modo geral, ao ser observada a variação de volume total e por terços, poucas diferenças foram encontradas 
entre as associações repetidas. Esse fato pode estar relacionado à metodologia adotada para alocação dos espécimes, quando a determinação do diâmetro inicial com limas manuais de calibre três vezes menor que o instrumento de preparo pareceu adequada para esse grupo dentário.

No presente estudo a porcentagem de áreas não instrumentadas foi significativamente afetada pela técnica de preparo, com valores que variaram de $12,52 \%$ a 56,93\%. A respeito dessa variável, com sistemas SAF, Reciproc, BioRace, TRUShape, Zuolo et al. (2018) obtiveram variação de 16,08\% a 32,38\%. Reciproc, WaveOne e BioRaCe foram testados no estudo de DeDeus et al. (2015) e também apresentam porcentagem de áreas não instrumentadas variando de 27,68\% a 60,77\%. Com base no exposto, pode-se afirmar que nenhum dos instrumentos supracitados foi capaz de tocar completamente as paredes dentinárias. A porcentagem de áreas não instrumentadas é influenciada pela anatomia interna e por fatores como design, cinemática e número de instrumentos utilizados (Baratto-Filho et al., 2009), como demonstrado aqui, melhores resultados estiveram associados à maior ampliação do canal radicular.

Como resultado da ação dos instrumentos utilizados no preparo dos canais radiculares, indesejavelmente ocorre a formação de debris, também observada neste estudo, mas sem diferenças significativas para porcentagem total e por terços em ambas as técnicas de preparo. De-Deus et al. (2015) reportaram os mesmos resultados, comparando os sistemas Reciproc, WaveOne e BioRaCe. Zuolo et al. (2018) também evidenciaram acúmulo de debris com o uso dos sistemas BioRace, Reciproc, SAF e TRUShape. Embora menor porcentagem de debris tenha sido observada quando da maior ampliação do canal radicular, a formação de debris, referida como a presença de um material com densidade semelhante à dentina em regiões previamente ocupadas por ar no interior do canal radicular não preparado, ocorre independente do sistema e técnica utilizada. A baixa porcentagem de debris está relacionada ao design do instrumento, da alteração no ângulo de corte e do alívio que continuamente muda sua direção de rotação durante sua ação, promovendo alteração de contato entre a superfície do instrumento e a parede do canal, o que facilita a sua remoção (van der Vyver et al., 2019).

Ainda pela análise de $\mu \mathrm{CT}$, consideradas as indicações do fabricante e os resultados encontrados por Webber (2016) e van der Vyver e Vorster (2017), que sugerem que o instrumento WP adapta-se à maioria dos canais radiculares, observou-se também uma variação de volume do grupo MF, após o uso de cada instrumento. Nesta avaliação pode-se verificar que o instrumento WP foi estatisticamente diferente dos demais nas comparações por terços e total ( $\mathrm{p}<0,05)$, corroborando os resultados citados anteriormente.

Neste estudo, ambas as técnicas promoveram variação de volume após o preparo, o que demonstrou eficácia do método adotado para alocação da amostra, com base na prática proposta por van der Vyver e Vorster (2017). Com relação às poucas diferenças encontradas, observa-se que embora haja um aumento progressivo no diâmetro dos instrumentos, há também compensação decrescente de taper ao longo da parte ativa. Desta forma, o diâmetro dos instrumentos torna-se aproximado nas mesmas regiões, revelando pouco desgaste de dentina. Esta característica de taper decrescente nos novos instrumentos favorece um preparo endodôntico mais conservador na região cervical. Assim, a escolha de instrumento do sistema WOG para o preparo endodôntico está relacionada ao D0, pois a maior variação de taper é observada de D0 a D5, o que pode justificar pontuais diferenças estatísticas entre os grupos estudados.

Por fim, com relação à variação de volume promovido pelo sistema WOG, melhores resultados estiveram associados ao modo MF, com indicações para associação de WS a WP. Entretanto, frente à anatomia dos incisivos inferiores, foi observada persistência de áreas não instrumentadas e presença de debris, sendo o desempenho de WS a WM, superior. 


\section{Conclusão}

Houve melhor desempenho dos instrumentos de maior calibre na variação do volume dos canais radiculares, bem como foi observada menor porcentagem de áreas não instrumentadas e de debris, quando da maior ampliação do canal radicular.

\section{Referências}

Alencar, A. H. G., Figueiredo, J. A. P., \& Estrela, C. (2008). Microtomografia computadorizada na avaliação do preparo do canal radicular: análise crítica. Robrac, 17(44), 159-165.

Baratto-Filho, F., Leonardi, D. P., Zielak, J. C., Vanni, J. R., Sayão-Maia, S. M. A., \& Sousa-Neto, M. D. (2009). Influence of protaper finishing files and sodium hypochlorite on cleaning and shaping of mandibuldar central incisors - a histological analysis. Journal of Applied Oral Science, 17(3), $229-233$.

Bueno, C., Oliveira, D. P., Pelegrine, R. A., Fontana, C. E., Rocha, D., \& Bueno, C. (2017). Fracture incidence of WaveOne and Reciproc files during root canal preparation of up to 3 posterior teeth: A prospective clinical study. Journal of Endodontics, 43(5), 705-708. https://doi.org/10.1016/j.joen.2016.12.024

Busquim, S., Cunha, R. S., Freire, L., Gavini, G., Machado, M. E., \& Santos, M. (2015). A micro-computed tomography evaluation of long-oval canal preparation using reciprocating or rotary systems. International Endodontic Journal, 48(10), 1001-1006. 10.1111/iej.12398

De-Deus, G., Marins, J., Neves, A., Reis, C., Fidel, S., Versiani, M. A., Alves, H., Lopes, R. T., \& Paciornik, S. (2014). Assessing accumulated hard-tissue debris using micro-computed tomography and free software for image processing and analysis. Journal of Endodontics, 40(2), 271-276. https://doi.org/10.1016/j.joen.2013.07.025

De-Deus, G., Belladonna, F. G., Silva, E. J., Marins, J. R., Souza, E. M., Perez, R., Lopes, R. T., Versiani, M. A., Paciornik, S., \& Neves, A. (2015). Micro-CT evaluation of non-instrumented canal areas with different enlargements performed by NiTi systems. Brazilian Dental Journal, $26(6)$, 624-629. https://doi.org/10.1590/0103-6440201300116

De-Deus, G., Belladonna, F. G., Zuolo, A. S., Cavalcante, D. M., Carvalhal, J. C. A., Simões-Carvalho, M., Souza, E. M., Lopes, R. T., \& Silva, E. J. N. L. (2019). XP-endo Finisher R instrument optimizes the removal of root filling remnants in oval-shaped canals. International Endodontic Journal, 52(6), 899907. https://doi.org/10.1111/iej.13077

Espir, C. G., Nascimento, C. A., Guerreiro-Tanomaru, J. M., Bonetti-Filho, I., \& Tanomaru-Filho, M. (2018). Radiographic and micro-computed tomography classification of root canal morphology and dentin thickness of mandibular incisors. Journal of Conservative Dentistry: JCD, $21(1), 57-62$.

Gergi, R., Arbab-Chirani, R., Osta, N., \& Naaman, A. (2014). Micro-computed tomographic evaluation of canal transportation instrumented by different kinematics rotary nickel-titanium instruments. Journal of Endodontics, 40(8), 1223-1227. https://doi.org/10.1016/j.joen.2014.01.039

Gergi, R., Osta, N., Bourbouze, G., Zgheib, C., Arbab-Chirani, R., \& Naaman, A. (2015). Effects of three nickel titanium instrument systems on root canal geometry assessed by micro-computed tomography. International Endodontic Journal, 48(2), 162-170. https://doi.org/10.1111/iej.12296

Guillén, R. E., Nabeshima, C. K., Caballero-Flores, H., Cayón, M. R., Mercadé, M., Cai, S., \& Machado, M. E. L. (2018). Evaluation of the WaveOne Gold and One Shape New Generation in reducing Enterococcus faecalis from root canal. Brazilian Dental Journal, 29(3), 249-253. https://doi.org/10.1590/01036440201801910

Guimarães, L. S., Gomes, C. C., Marceliano-Alves, M. F., Cunha, R. S., Provenzano, J. C., \& Siqueira, J. F., Jr (2017). Preparation of Oval-shaped canals with TRUShape and Reciproc systems: A micro-computed tomography study using contralateral premolars. Journal of Endodontics, 43(6), 1018-1022. https://doi.org/10.1016/j.joen.2017.01.028

Hülsmann, M., Rümmelin, C., \& Schäfers, F. (1997). Root canal cleanliness after preparation with different endodontic handpieces and hand instruments: a comparative SEM investigation. Journal of Endodontics, 23(5), 301-306. https://doi.org/10.1016/S0099-2399(97)80410-4

Langeland, K., Liao, K., \& Pascon, E. A. (1985). Work-saving devices in endodontics: efficacy of sonic and ultrasonic techniques. Journal of Endodontics, 11(11), 499-510. https://doi.org/10.1016/s0099-2399(85)80223-5

Lim, Y. J., Park, S. J., Kim, H. C., \& Min, K. S. (2013). Comparison of the centering ability of Wave-One and Reciproc nickel-titanium instruments in simulated curved canals. Restorative Dentistry \& Endodontics, 38(1), 21-25. https://doi.org/10.5395/rde.2013.38.1.21

Lorencetti, K. T., Silva-Sousa, Y. T. C., Nascimento, G. E., Messias, D. C. F., Colucci, V., Rached-Junior, F. A., \& Silva, S. R. C. (2014). Influence of apical enlargement in cleaning of curved canals using negative pressure system. Brazilian Dental Journal, 25(5), 430-434. https://doi.org/10.1590/01036440201302435

Milanezi de Almeida, M., Bernardineli, N., Ordinola-Zapata, R., Villas-Bôas, M. H., Amoroso-Silva, P. A., Brandão, C. G., Guimarães, B. M., Gomes de Moraes, I., \& Húngaro-Duarte, M. A. (2013). Micro-computed tomography analysis of the root canal anatomy and prevalence of oval canals in mandibular incisors. Journal of Endodontics, 39(12), 1529-1533. https://doi.org/10.1016/j.joen.2013.08.033

Neves, M. A., Provenzano, J. C., Rôças, I. N., \& Siqueira, J. F., Jr (2016). Clinical antibacterial effectiveness of root canal preparation with reciprocating single-instrument or continuously rotating multi-instrument systems. Journal of Endodontics, 42(1), 25-29. https://doi.org/10.1016/j.joen.2015.09.019

Nielsen, R. B., Alyassin, A. M., Peters, D. D., Carnes, D. L., \& Lancaster, J. (1995). Microcomputed tomography: an advanced system for detailed endodontic research. Journal of Endodontics, 21(11), 561-568. https://doi.org/10.1016/S0099-2399(06)80986-6 
Paqué, F., Laib, A., Gautschi, H., \& Zehnder, M. (2009). Hard-tissue debris accumulation analysis by high-resolution computed tomography scans. Journal of Endodontics, 35(7), 1044-1047. 10.1016/j.joen.2009.04.026

Paqué, F., \& Peters, O. A. (2011) Micro-computed tomography evaluation of the preparation of long oval root canals in mandibular molars with the selfadjusting file. Journal of Endodontics, 37(4), 517-521. 10.1016/j.joen.2010.12.011

Plotino, G., Grande, N. M., Pecci, R., Bedini, R., Pameijer, C. H., \& Somma, F. (2006). Three-dimensional imaging using microcomputed tomography for studying tooth macromorphology. Journal of the American Dental Association (1939), 137(11), 1555-1561.

Plotino, G., Özyürek, T., Grande, N. M., \& Gündoğar, M. (2019). Influence of size and taper of basic root canal preparation on root canal cleanliness: a scanning electron microscopy study. International Endodontic Journal, 52(3), 343-351. https://doi.org/10.1111/iej.13002

Robinson, J. P., Lumley, P. J., Claridge, E., Cooper, P. R., Grover, L. M., Williams, R. L., \& Walmsley, A. D. (2012). An analytical Micro CT methodology for quantifying inorganic dentine debris following internal tooth preparation. Journal of Dentistry, 40(11), 999-1005. https://doi.org/10.1016/j.jdent.2012.08.007

Schilder H. (1974). Cleaning and shaping the root canal. Dental Clinics of North America, 18(2), 269-296.

Sousa-Neto, M. D., Silva-Sousa, Y. C., Mazzi-Chaves, J. F., Carvalho, K. K. T., Barbosa, A. F. S., Versiani, M. A., Jacobs, R., \& Leoni, G. B. (2018). Root canal preparation using micro-computed tomography analysis: a literature review. Brazilian Oral Research, 32(Sup. 1), e66. https://doi.org/10.1590/18073107bor-2018.vol32.0066

Tambe, V. H., Nagmode, P. S., Abraham, S., Patait, M., Lahoti, P. V., \& Jaju, N. (2014). Comparison of canal transportation and centering ability of rotary protaper, one shape system and wave one system using cone beam computed tomography: An in vitro study. Journal of Conservative Dentistry: JCD, 17(6), 561-565. https://doi.org/10.4103/0972-0707.144605

van der Vyver, P. J., \& Vorster, M. (2017). WaveOne ${ }^{\circledR}$ Gold reciprocating instruments: clinical application in the private practice: Part 1. International Dentistry - African Edition, 7(4), 6-19.

van der Vyver, P. J., Paleker, F., Vorster, M., \& de Wet, F. A. (2019). Root canal shaping using nickel titanium, m-wire, and gold wire: A micro-computed tomographic comparative study of One Shape, ProTaper Next, and WaveOne Gold instruments in maxillary first molars. Journal of Endodontics, 45(1), 62-67. https://doi.org/10.1016/j.joen.2018.09.013

Velozo, C., Silva, S., Almeida, A., Romeiro, K., Vieira, B., Dantas, H., Sousa, F., \& De Albuquerque, D.S. (2020). Shaping ability of XP-endo Shaper and ProTaper Next in long oval-shaped canals: a micro-computed tomography study. International Endodontic Journal, 53(7), 998-1006. https://doi.org/10.1111/iej.13301

Versiani, M. A., Pécora, J. D., \& de Sousa-Neto, M. D. (2011). Flat-oval root canal preparation with self-adjusting file instrument: a micro-computed tomography study. Journal of Endodontics, 37(7), 1002-1007. https://doi.org/10.1016/j.joen.2011.03.017

Versiani, M. A., Carvalho, K., Mazzi-Chaves, J. F., \& Sousa-Neto, M. D. (2018). Micro-computed tomographic evaluation of the shaping ability of XP-endo Shaper, iRaCe, and EdgeFile systems in long oval-shaped canals. Journal of Endodontics, 44(3), 489-495. https://doi.org/10.1016/j.joen.2017.09.008

Versiani, M. A., Alves, F. R., Andrade-Junior, C. V., Marceliano-Alves, M. F., Provenzano, J. C., Rôças, I. N., Sousa-Neto, M. D., \& Siqueira, J. F., Jr (2016). Micro-CT evaluation of the efficacy of hard-tissue removal from the root canal and isthmus area by positive and negative pressure irrigation systems. International Endodontic Journal, 49(11), 1079-1087. https://doi.org/10.1111/iej.12559

Villas-Bôas, M. H., Bernardineli, N., Cavenago, B. C., Marciano, M., Del Carpio-Perochena, A., de Moraes, I. G., Duarte, M. H., Bramante, C. M., \& Ordinola-Zapata, R. (2011). Micro-computed tomography study of the internal anatomy of mesial root canals of mandibular molars. Journal of Endodontics, 37(12), 1682-1686. https://doi.org/10.1016/j.joen.2011.08.001

Webber, J. (2015). Shaping canals with confidence: WaveOne GOLD single-file reciprocating system. Roots, 6(3), 34-40.

Wu, M. K., R'oris, A., Barkis, D., \& Wesselink, P. R. (2000). Prevalence and extent of long oval canals in the apical third. Oral Surgery, Oral Medicine, Oral Pathology, Oral Radiology, and Endodontics, 89(6), 739-743. https://doi.org/10.1067/moe.2000.106344

Yared, G. (2008). Canal preparation using only one Ni-Ti rotary instrument: preliminary observations. International Endodontic Journal, 41(4), 339-344. $10.1111 / \mathrm{j} .1365-2591.2007 .01351 . \mathrm{x}$

Zuolo, M. L., Zaia, A. A., Belladonna, F. G., Silva, E., Souza, E. M., Versiani, M. A., Lopes, R. T., \& De-Deus, G. (2018). Micro-CT assessment of the shaping ability of four root canal instrumentation systems in oval-shaped canals. International Endodontic Journal, 51(5), 564-571. https://doi.org/10.1111/iej.12810 